\title{
EQUILÍBRIO LÍQUIDO-LÍQUIDO DO SISTEMA PSEUDOBINÁRIO CONTENDO ÓLEO DE SOJA + (ETANOL + ÁGUA) FRENTE A DIVERSAS TEMPERATURAS
}

\author{
J. L. A. DAGOSTIN ${ }^{1}$, D. CARPINE ${ }^{1}$, L. P. RAMOS ${ }^{2}$, M. R. MAFRA ${ }^{1}$ e M. L. CORAZZA ${ }^{1}$ \\ ${ }^{1}$ Universidade Federal do Paraná, Departamento de Engenharia Química \\ ${ }^{2}$ Universidade Federal do Paraná, Departamento de Química \\ E-mail para contato: corazza@ufpr.br
}

\begin{abstract}
RESUMO - Solventes alternativos vêm sendo buscados para a obtenção de óleos vegetais comestíveis devido ao aumento nas exigências por produtos de alta qualidade por consumidores finais bem como necessidades ambientais. $\mathrm{O}$ etanol é um solvente renovável de grande disponibilidade no Brasil. Na literatura, vários estudos encontramse relatados sobre a solubilidade e a capacidade de extração do óleo de soja por etanol e em frações (etanol + água), porém, estes dados são investigados numa faixa pequena de temperaturas de trabalho. $\mathrm{O}$ objetivo deste trabalho foi verificar a solubilidade do pseudobinário óleo de soja + (etanol + água) frente a diversas temperaturas e em diferentes teores de água no etanol (solvente) pelo método de ponto de névoa. A concentração de água e baixas temperaturas promoveram a diminuição da solubilidade do sistema.
\end{abstract}

\section{INTRODUÇÃO}

Com a crescente demanda por cadeias produtivas seguras e ambientalmente limpas, muitas pesquisas vêm sendo realizadas no sentido da readequação de processos industriais, como a substituição ou diminuição do uso de fontes não renováveis, como derivados do petróleo. O processo de extração de óleo de soja pode ser descrito como um destes processos. O método de extração baseia-se no arraste dos componentes oleosos por uma mistura de hidrocarbonetos derivados do petróleo, muitas vezes denominada hexano comercial.

A versatilidade do uso do hexano está na sua alta eficiência em solubilizar óleos e gorduras, além de possuir ponto de evaporação entre 65 e $69^{\circ} \mathrm{C}$ (Kemper, 2005). No entanto, ela é altamente volátil, podendo formar misturas explosivas com o ar. Além disso, este solvente pode ser tóxico se inalado ou ingerido em concentrações significativas. Segundo o U.S. Occupational Safety and Health Administration, o limite diário de exposição ao hexano para quem trabalha sujeito a seu contato é de $500 \mathrm{ppm}$, sendo recomendado um máximo de $50 \mathrm{ppm}$. Não existem normas regulamentadoras para o consumo de hexano em alimentos, embora o U.S. Environmental Protection Agency tenha estimado que o consumo de menos de 0,06 mg hexano por kilograma de massa corpórea seja provavelmente seguro (Osha, 2012; Hammond et al., 2005; Kemper, 2005).

Dentre as alternativas ao uso de hexano para a extração de óleo vegetal, destaca-se o uso de tricloroetileno, etanol, isopropanol, propanol, entre outros (Thomas, 2003; Seth et al., 2007; Gandhi et al., 2003). Dentre os solventes estudados, o etanol parece ser um solvente promissor para indústrias brasileiras, uma vez que o país é o segundo maior produtor mundial de etanol e seu 
custo de produção é relativamente baixo comparado a outros países. Além disso, ele apresenta menores riscos a saúde e a segurança, pois é atóxico e apresenta menor inflamabilidade comparado à hexana (Hammond et al., 2005; Kemper, 2005).

O etanol é completamente miscível com água, sendo capaz de formar um azeótropo a frações molares de aproximadamente 89 mols de etanol para 11 mols de água ou, em termos volumétricos, aproximadamente $96 \%$ (vol.) de etanol para 4\% (vol.) de água (Ferreira et al., 2004; Lei et al., 2002). O problema da presença de água no etanol é que ela reduz drasticamente a solubilização do óleo na mistura, e consequentemente, reduz a eficiência da extração (Gandhi et al., 2003). Por formar uma mistura azeotrópica, o custo de obtenção de etanol absoluto (anidro) torna-se elevado por depender de técnicas subsequentes à destilação.

Dentro deste contexto, é de relevante importância conhecer a solubilidade do etanol em óleo de soja a diferentes graus de hidratação. Assim, neste estudo verificou-se a solubilidade do óleo de soja em etanol com diferentes teores de água por meio da obtenção de dados de equilíbrio líquidolíquido em diagramas de fase a temperaturas de 5 a $75^{\circ} \mathrm{C}$.

\section{MATERIAL E MÉTODOS}

\subsection{Material}

Ensaios de solubilidade: Utilizou-se óleo de soja refinado (Liza), etanol anidro e hidratado 96\% vol (Panreac).

Determinação da composição dos sistemas: Fez-se o uso de acetato de etila (F.Maia), reagente de Karl Fisher (Panreac) e metanol anidro (Panreac).

Todos os reagentes e matérias-primas foram utilizados como recebidos.

\subsection{Métodos}

Determinação das curvas de solubilidade: Os dados de equilíbrio líquido-líquido (ELL) foram obtidos em células de equilíbrio conforme descrito por (da Silva et al., 2013, Kanda et al., 2013). A temperatura das células foi monitorada por indicador de temperatura Watlow SD31 com sensor PT100 $\left( \pm 0.1^{\circ} \mathrm{C}\right)$ e controlada por banho ultratermostático (Nova Ética 521/D). Os dados de ELL foram determinados dentro da faixa de 5 a $75{ }^{\circ} \mathrm{C}$ variando-se $5{ }^{\circ} \mathrm{C}$ para cada medida. As curvas de solubilidade do pseudobinário óleo de soja + (etanol + água) foram feitas utilizando-se etanol absoluto e $96 \%$ (vol), além de suas misturas com água tipo II para obter soluções etanólicas com diferentes níveis de hidratação. Assim, foram realizados ensaios de solubilidade com misturas etanol + água nas proporções mássicas: 99,93\% (absoluto), 98\%, 96\%, 94,15\% e 90\%. A concentração de todas as soluções alcoólicas e do óleo foi confirmada por Karl-Fisher. A homogeneidade de fases do sistema foi verificada pelo método do ponto de névoa utilizando-se duas células de solubilidade, onde em cada uma a titulação iniciou-se ou com óleo de soja ou com (etanol + água) em excesso. A titulação ocorreu simultaneamente em ambas as células para cada temperatura avaliada.

Determinação do teor de água: O conteúdo de água dos reagentes, das misturas (etanol + água) e do óleo de soja foi avaliado por titulação de Karl Fischer (Mettler-Toledo modelo V30) 
pelo método oficial Ca 2e-84 da AOCS (AOCS, 2001).

\section{RESULTADOS E DISCUSSÃO}

As curvas de solubilidade para o sistema proposto frente à variação de temperatura podem ser verificadas na Figura 1. Conforme esperado, a concentração de água influenciou de forma expressiva e negativa a solubilidade do etanol no óleo de soja. A baixas temperaturas $\left(5\right.$ a $\left.15{ }^{\circ} \mathrm{C}\right)$ houve uma maior dificuldade em solubilizar o sistema devido à maior viscosidade do óleo e à menor energia disponível. Além disso, quanto menor a temperatura, maiores gotas foram formadas, o que dificulta a transferência de moléculas entre os componentes.

No trabalho de Follegatti-Romero et al. (2010) foi realizado estudo de solubilidade para o sistema binário óleo de soja + etanol anidro na faixa de temperatura de 25 a $60{ }^{\circ} \mathrm{C}$. Estes dados experimentais estão presentes na Figura 1(A). Os autores aplicaram um modelo NRTL e encontraram para este sistema uma temperatura crítica de solução extrapolada de $69,1^{\circ} \mathrm{C}$. No presente trabalho, ao utilizar etanol absoluto, encontrou-se experimentalmente que acima de $64{ }^{\circ} \mathrm{C}$ ocorreu miscibilidade completa do sistema, ou seja, cerca de $5{ }^{\circ} \mathrm{C}$ abaixo da temperatura predita pelo modelo ajustado pelo referido autor. Esta diferença foi atribuída ao fato do modelo utilizado não prever um "achatamento" da curva próximo à sua inflexão, o qual foi encontrado experimentalmente neste trabalho.

As misturas óleo de soja + (etanol + água) apresentaram maior região de solubilidade nas frações ricas em óleo. Nas frações ricas em etanol 90\% (Figura 1(E)) a capacidade de solubilização diminuiu a temperaturas de 20 a $40{ }^{\circ} \mathrm{C}$, de tal forma que experimentalmente tornouse impossível a coleta dos pontos de mudança de fase. 

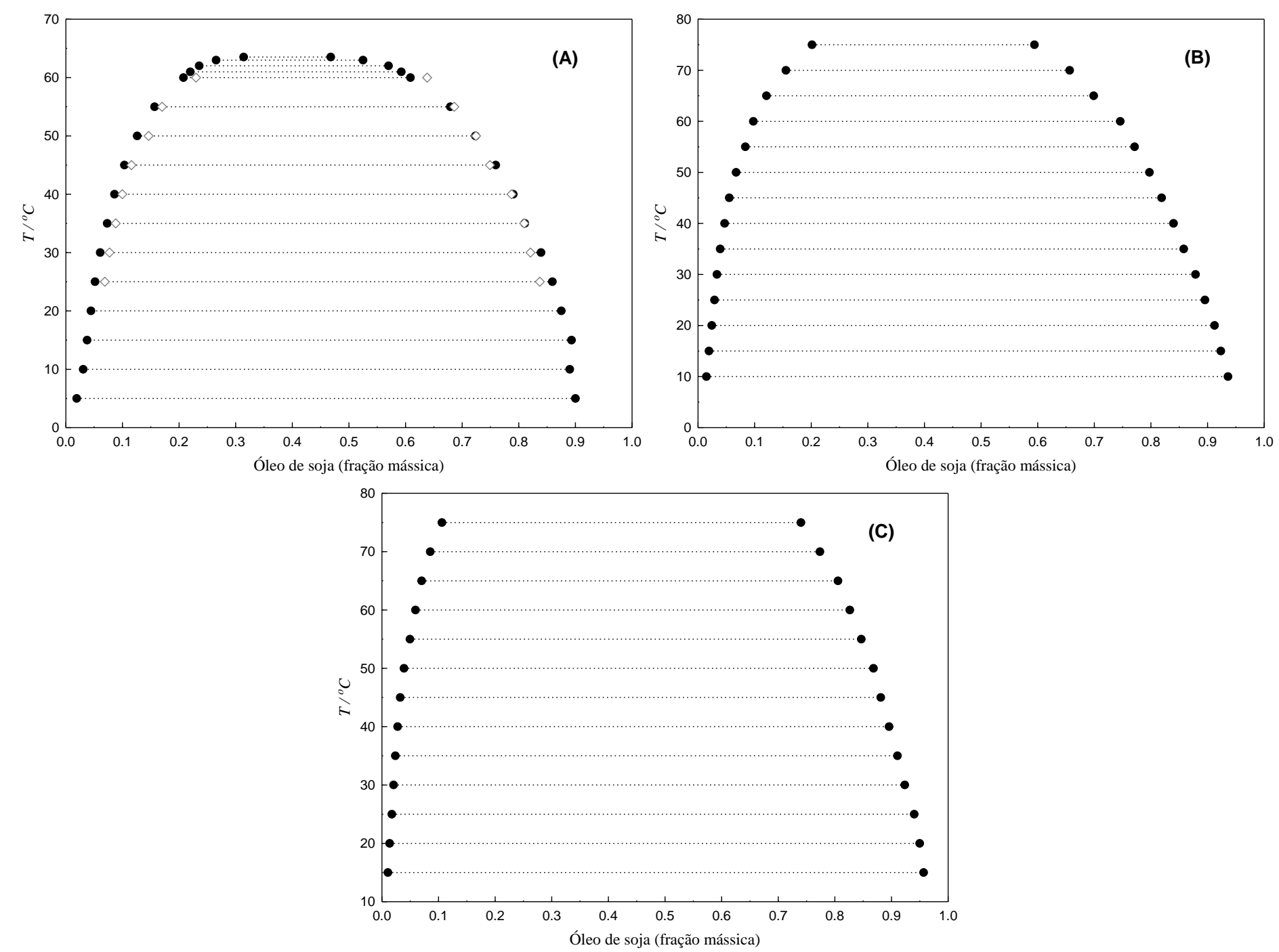

Figura 1 - Diagrama $T$ - $x$ dos sistemas (A) óleo de soja + etanol anidro $(99,8 \%)$, (B) óleo de soja + etanol 98\%, e (C) óleo de soja + etanol $96 \%$ (massa). Linhas de amarração experimentais (- , este trabalho; $\diamond$, Follegatti-Romero et al., 2010). 

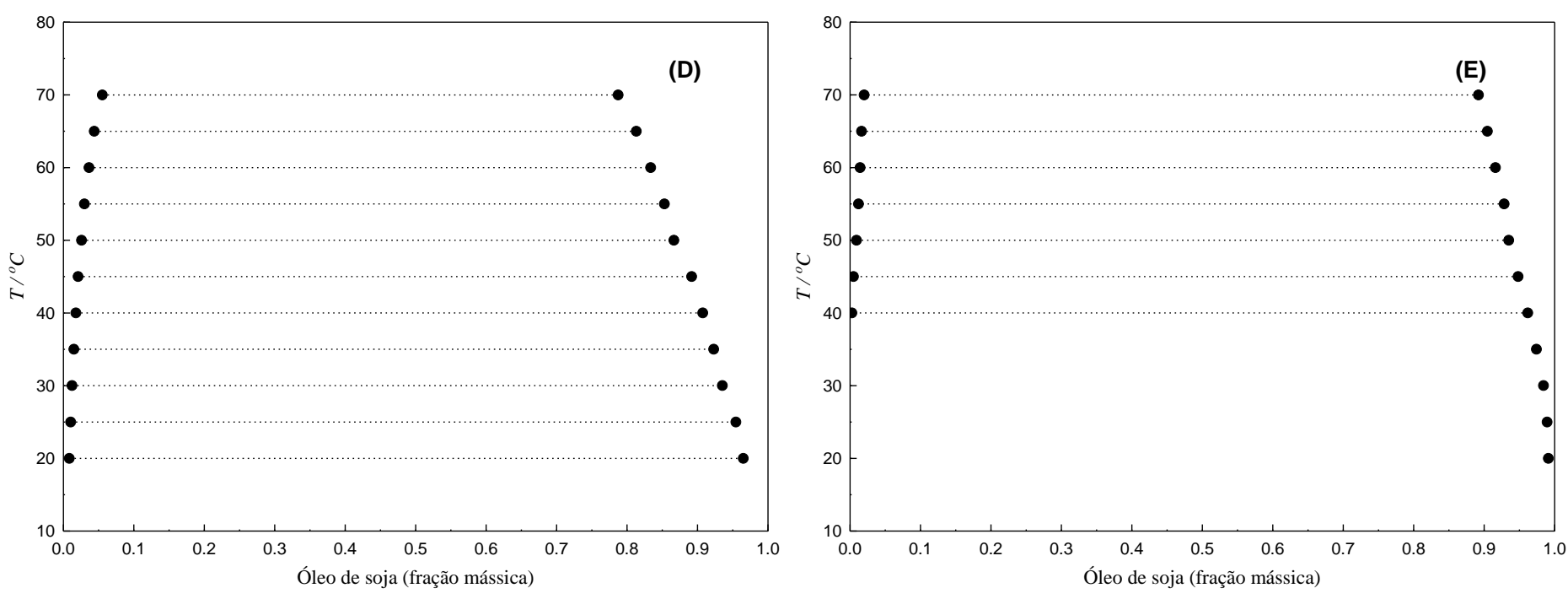

Figura 1 (continuação) - Diagrama T-x dos sistemas (D) óleo de soja + etanol 94,15\%, e (E) óleo de soja + etanol 90\% (massa). Linhas de amarração experimentais, - 
Os resultados destes sistemas podem ser úteis na predição da capacidade de extração de óleos por etanol. A água é um componente presente nas matrizes utilizadas para a extração de óleos, em maior ou menor quantidade. Por isso, mesmo que seja feito uso de etanol anidro num processo de extração de óleo, deve-se levar em conta a transferência de água ao sistema. Desta forma, justifica-se o estudo da solubilidade do sistema óleo de soja + (etanol + água) à variabilidade do teor de água, pois este é decorrente dos parâmetros do processo e da quantidade de água da matriz.

Enquanto houver solubilidade do sistema óleo + etanol hidratado, é factível que haja a capacidade de extração de óleo da matriz de sua origem. Para que isso ocorra a condições normais de pressão, é necessário que o sistema apresente alguma solubilidade a temperaturas abaixo do ponto de ebulição do azeótropo etanol + água (cerca de $78^{\circ} \mathrm{C}$ para misturas de 80 a $99 \%$ ). Além disso, ajustes na proporção do solvente alcoólico devem ser realizados para permanecer na faixa de solubilidade dos componentes.

\section{CONCLUSÃO}

Neste trabalho, foram apresentados dados de equilíbrio liquido-líquido para sistemas pseudobinários contendo óleo de soja + (etanol + água) a diferentes temperaturas. Com o aumento da temperatura, todos os sistemas apresentaram um aumento de solubilidade. Com o incremento da concentração de água ao sistema, a solubilidade total diminuiu. Os dados de solubilidade aqui apresentados podem ser úteis no projeto e otimização da extração de óleo vegetal por etanol e etanol + água. É provável ainda, que ao se utilizar outros tipos de óleo vegetal seja possível obter comportamentos similares sobre a solubilidade deste tipo de sistema, principalmente se o óleo utilizado possuir propriedades físicas e/ou químicas similares ao óleo de soja. Levando-se em conta que em processos de extração é comum utilizar-se maior quantidade de solvente em relação ao soluto, a condições normais de pressão a extração de óleo de soja por etanol hidratado não deverá ocorrer a concentrações de álcool muito menores que $90 \%$.

\section{Agradecimentos}

Os autores agradecem a CAPES, CNPq, PRH24 e Fundação Araucária-Paraná pelo auxílio financeiro e bolsa concedida.

\section{REFERÊNCIAS}

AOCS, Official Methods and Recommended Practices of the American Oil Chemists' Society, $5^{\mathrm{a}}$ ed., Champaign: AOCS Press, 2001.

DA SILVA, D. I. S.; MAFRA, M. R.; DA SILVA, F. R.; NDIAYE, P. M.; RAMOS, L. P.; CARDOZO FILHO, L.; CORAZZA, M. L. Liquid-liquid and vapor-liquid equilibrium data for biodiesel reaction-separation systems. Fuel, v. 108 p. 269-276, 2013.

FERREIRA, S. M.; DE OLIVEIRA, E.; DUARTE, U. Comportamento da gasolina com etanol (E20) e da gasolina pura após a simulação de um vazamento em colunas de laboratório. Geologia USP. Série Científica, v. 4, n. 2, p. 91-102, 2004.

FOLlEGATTI-ROMERO, L. A.; LANZA, M.; DA SILVA, C. A. S.; BATISTA, E. A. C.; MEIRELLES, A. J. A. Mutual solubility of pseudobinary systems containing vegetable oils 
and anhydrous ethanol from (298.15 to 333.15) K. J. Chem. Eng. Data, v. 55(8), p. 2750 2756, 2010.

GANDHI, A. P.; JOSHI, K. C.; JHA, K.; PARIHAR, V. S.; SRIVASTAV, D. C.; RAGHUNADH, P.; KAWALKAR, J.; JAIN, S. K.; TRIPATHI, R. N. Studies on alternative solvents for the extraction of oil soybean. Int. J. of Food Sci. and Tech., v. 38, p. 369-375, 2003.

HAMMOND, E. G.; JOHNSON, L. A.; SU, C.; WANG, T.; WHITE, P. J. Soybean oil. Em: SHAHIDI, F. (ed.) Bailey's industrial oil and fat products, $6^{\mathrm{a}}$ ed. Nova York: WileyInterscience, 2005.

KANDA, L. R.; VOLL, F. A. P.; CORAZZA, M. L. LLE for the systems ethyl palmitate (palmitic acid)(1) + ethanol(2) + glycerol (water)(3). Fluid Phase Equilib., v. 354, p. 147-155, 2013.

KEMPER, T. G. Oil Extraction. Em: SHAHIDI, F. (ed) Bailey's Industrial Oil and Fat Products, $6^{\mathrm{a}}$ ed. Nova York: Wiley-Interscience, 2005.

LEI, Z.; WANG, H.; ZHOU, R.; DUAN, Z. Influence of salt added to solvent on extractive distillation. Chem. Eng. J., v. 87(2), p. 149-156, 2002.

OSHA - Occupational Safety and Health Guideline for n-Hexane. Disponível em: <https://www.osha.gov/dts/chemicalsampling/data/CH_245400.html>. Acesso em: 09/04/2014.

SETH, S.; AGRAWAL, Y. C.; GHOSH, P. K.; JAYAS, D. S.; SINGH, B. P. N. Oil extraction rates of soya bean using isopropyl alcohol as solvent. Biosystems Eng., v. 97(2), p. 209-217, 2007.

THOMAS, G. C. Análise Teórico-Experimental da Extração de Óleo de Soja em Instalação Industrial do Tipo Rotocell. 125 f. Tese (Doutorado em Engenharia Mecânica) - Programa de Pós-graduação em Engenharia Mecânica, Universidade Federal do Rio Grande do Sul, Porto Alegre, 2003. 